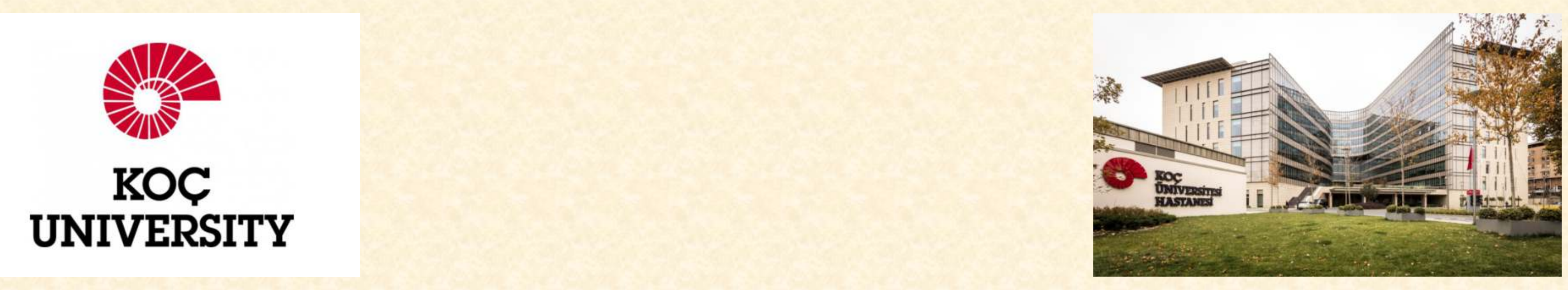

\title{
THE ROLE OF LIVER RESECTION IN PRIMARY, SECONDARY AND TERTIARY SETTING FOR THE SURGICAL TREATMENT OF OVARIAN CANCER
}

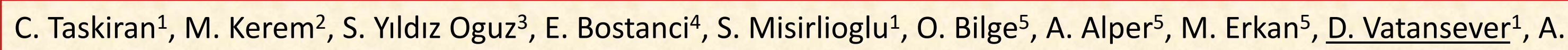
Onan ${ }^{4}$, M. Arvas ${ }^{1}$, H. Güner ${ }^{4}$.

${ }^{1}$ Koc University, Department of Obstetrics and Gynecology- Gynecologic Oncology Division, Istanbul, Turkey.

${ }^{2}$ Gazi University, Department of General Surgery, Ankara, Turkey.

${ }^{3}$ Koc University, Department of Obstetrics and Gynecology, Istanbul, Turkey.

${ }^{4}$ Gazi University, Department of Obstetrics and Gynecology, Ankara, Turkey.

${ }^{5}$ Koc University, Department of General Surgery, Istanbul, Turkey.

\begin{abstract}
Background and Aims:
The aim of the current study was to evaluate the role of liver resection for cytoreductive surgery (CRS) of ovarian cancer (OC) in primary, secondary and tertiary setting.

\section{Methods:}

All patients with $O C$ who underwent liver resection as a part of primary, secondary or tertiary CRS between $01 / 2006$ and 12/2018 at Gazi University, VKF Koc University and American Hospital were retrospectively reviewed. Data retrieved from the special gynecologic oncology records, and surgico-pathological electronical database.

Results:

A total of 67 patients were analyzed with a median age of 61 years. The resection was performed at primary cytoreduction in 30 patients $(45 \%)$, at secondary in $29(43 \%)$, and at tertiary in 8 patients $(12 \%)$. Two patients had left hepatectomy, 2 had right hepatectomy, 3 had partial hepatectomy, 3 had caudate lobectomy, 26 had unisegmentectomy, 4 'had multiple segmentectomy, and 27 had metastasectomy. Metastasectomy and segmentectomy rates were similar in primary And recurrent settings whereas hepatectomy were performed only in recurrent cases. Further surgical procedures required to achieve maximal cytoreduction in addition to liver resection: bowel resection in 24 , splenectomy in 11 , diaphragmatic resection in 11, distal pancreatectomy in 2, cholecystectomy in 7 , and partial gastric resection in 2 patients. Estimated blood loss was $470 \mathrm{~mL}$ (150-1350). The only post-operative complication was a sub-hepatic abscess in one of the patients. Intraoperative and postoperative minor or major complication rates did not differ between primary and recurrent cytoreduction groups. The median overall survival was 42 (2 -156) months in patients with liver resection.
\end{abstract}

\section{Conclusions:}

Resection of liver metastases of ovarian cancer is safe and feasible both in primary and recurrent settings when it is performed by experienced surgeons in specialized dedicated oncological centers.

\section{Keywords:}

Ovarian Cancer, Liver Metastases, Cytoreductive Surgery, Liver Resection

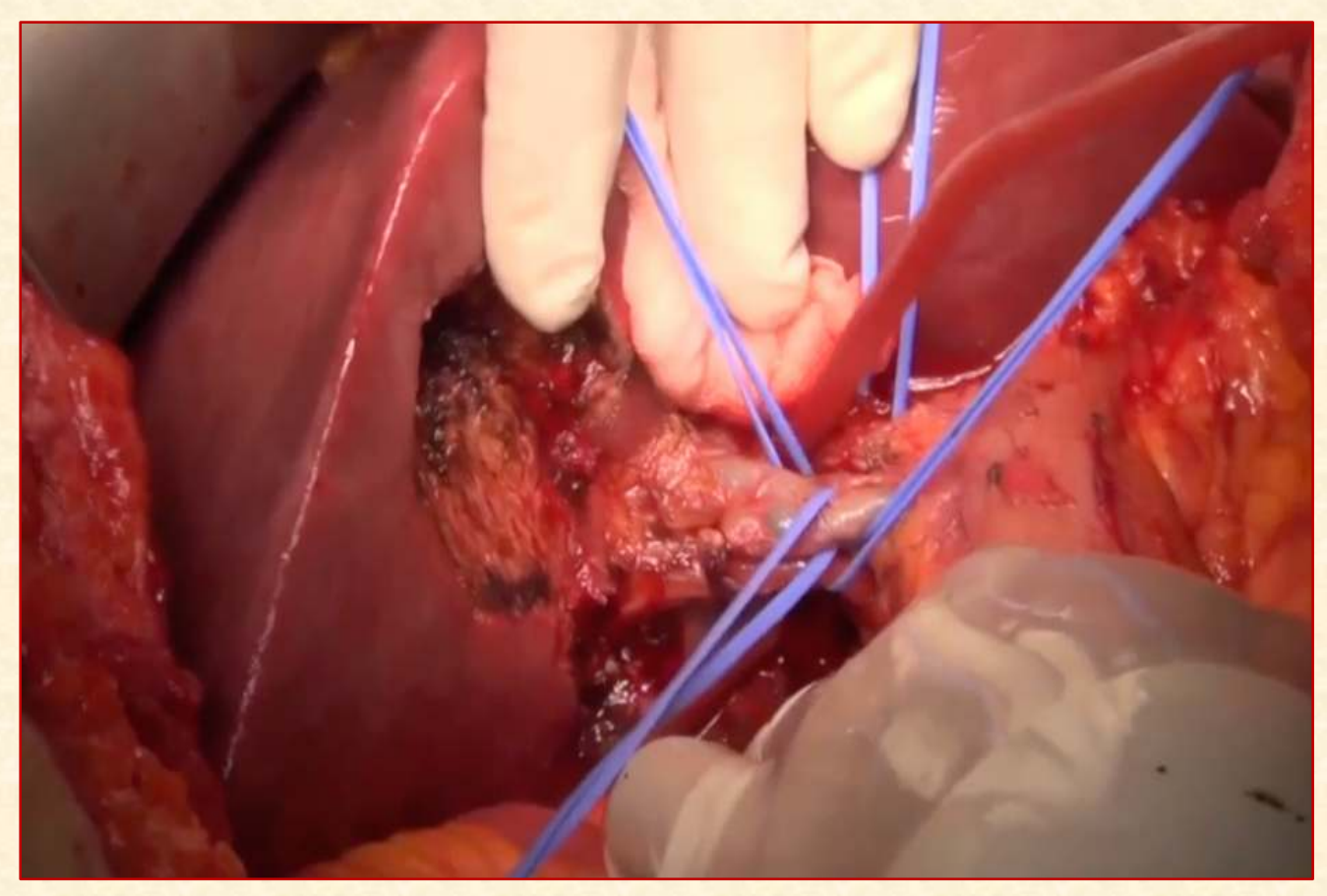

